\title{
Rare fibrosing granulomatous mediastinitis of tuberculosis with involvement of the transverse sinus
}

Chong Zhang, MD, ${ }^{a}$ Minhua Yao, MD, ${ }^{\mathrm{b}}$ Zhefeng $\mathrm{Yu}, \mathrm{MD},{ }^{\mathrm{c}}$ Lijun Jiang, MD, ${ }^{\mathrm{a}}$ Xiaoying Jiang, MD, and Yiming $\mathrm{Ni}, \mathrm{MD}^{\mathrm{a}}{ }^{\mathrm{a}}$ Hangzhou, China

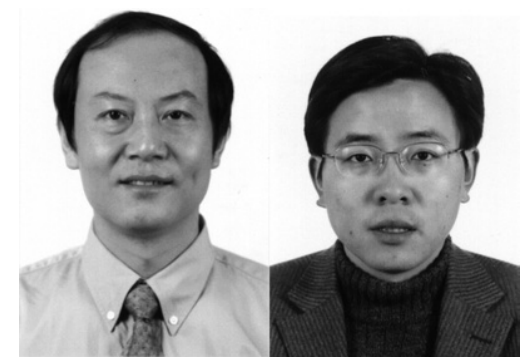

Professor and Dr Ni, and Dr Zhang (left to right)
$\mathrm{F}$ ibrosing granulomatous mediastinitis is rarely encountered; moreover, vascular narrowing or obstruction owing to this process is an uncommon condition, with involvement of the pulmonary artery, pulmonary veins, and coronary artery usually resulting in clinical manifestations. A case of narrowing of these vessels owing to fibrosing mediastinitis is reported.

\section{Clinical Summary}

A 63-year-old man was admitted to the hospital with reports of intermittent dyspnea and palpitation for 3 years. No abnormalities were evident on blood tests. The electrocardiogram showed premature atrial beats. The chest radiograph revealed mild elevation of the left bronchus. Echocardiography showed a large capsular space around the roots of the aorta and main pulmonary artery. The magnetic resonance imaging scan showed a giant mass near the aorta, main pulmonary artery, and left atrium, which severely compressed the vessels. The computed tomographic angiogram confirmed the

From the Department of Thoracic and Cardiovascular Surgery, The First Affiliated Hospital, ${ }^{\mathrm{a}}$ the Department of TCM, The Children's Hospital, ${ }^{\mathrm{b}}$ and the Department of Radiology, The First Affiliated Hospital, ${ }^{c}$ College of Medicine, Zhejiang University, Hangzhou, China.

Received for publication Sept 20, 2006; accepted for publication Nov 8 , 2006.

Address for reprints: Yiming Ni, MD, Department of Thoracic and Cardiovascular Surgery, The First Affiliated Hospital, College of Medicine, Zhejiang University, Hangzhou 310003, China (E-mail: haiyanzhangchong@ 163.com).

J Thorac Cardiovasc Surg 2007;133:836-7

$0022-5223 / \$ 32.00$

Copyright @ 2007 by The American Association for Thoracic Surgery doi:10.1016/j.jtcvs.2006.11.023 giant mediastinal mass with dimensions of 7.5 $\times 7.0 \times 6.5 \mathrm{~cm}$ in the transverse sinus involving the aorta, pulmonary artery, pulmonary veins, and coronary artery (Figure 1 and 2, A). Seven days later, the patient underwent surgery.

After the chest was opened through a median sternotomy, the pericardium appeared adhesive and very tense, particularly consistently tense and hard around the roots of aorta and main pulmonary artery. The pericardium was separated, starting from the right ventricle where the surface was less tense. After an incision between the aorta and main pulmonary artery was made, a great deal of flavescent viscous liquid with caseous necrosis flowed out of the mass from the incision (Figure 2, B). The encapsulated wall, which was toughly adherent to the aorta, pulmonary artery, and left atrium, was very thick and hard. The surgeon could not completely excise the wall of mass. A tube was placed into the cavity for drainage.

The patient had an uneventful postoperative recovery and was discharged on the seventh postoperative day. The pathologic report showed the mass was fibrous granuloma of tuberculosis. On follow-up, the patient was well without any reports such dyspnea, cough, or palpitation. The body temperature was normal and he constantly took antituberculosis drugs. The postoperative computed tomographic angiogram on a follow-up visit showed that the dimensions of cavity had obviously decreased and the narrowing of pulmonary artery, pulmonary veins, and coronary artery had disappeared.

\section{Discussion}

Mediastinal granuloma is the term used for caseous or fibrocaseous nodes or masses found in the mediastinum. Tuberculosis is an important cause of mediastinal granuloma with fibrosing mediastinitis. ${ }^{1}$ Fibrosing mediastinitis is a chronic process and a late

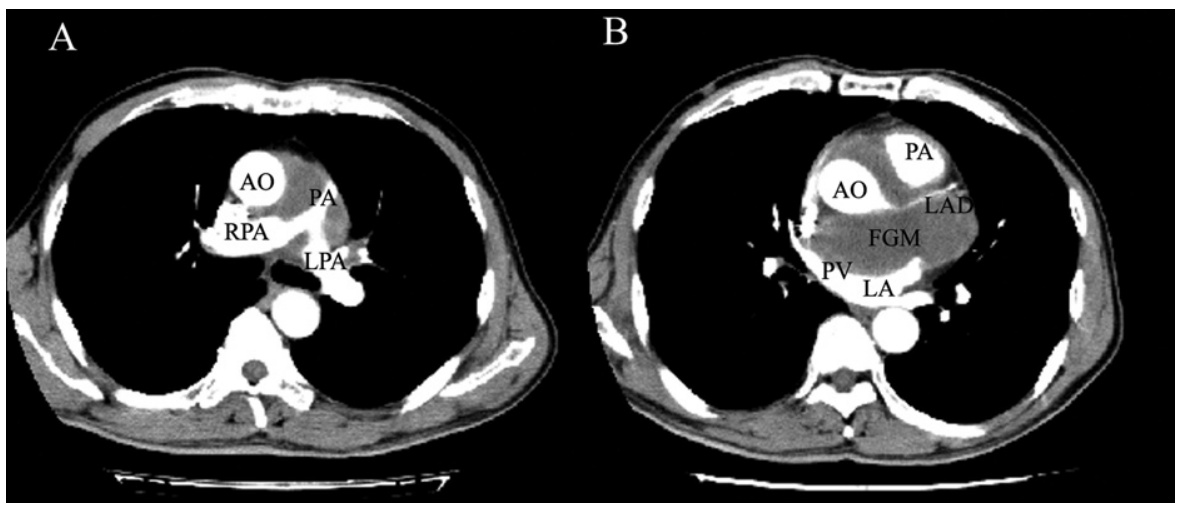

Figure 1. Preoperative computed tomographic angiogram showed a mediastinal mass compressing the vessels (A), narrowing the pulmonary artery (B), and narrowing the pulmonary veins and coronary artery. $A O$, Aorta; $P A$, pulmonary artery; $L P A$, left pulmonary artery; $R P A$, right pulmonary artery; $F G M$, fibrosing granulomatous mass; $L A D$, left anterior descending; $L A$, left atrium, $P V$, pulmonary vein. 


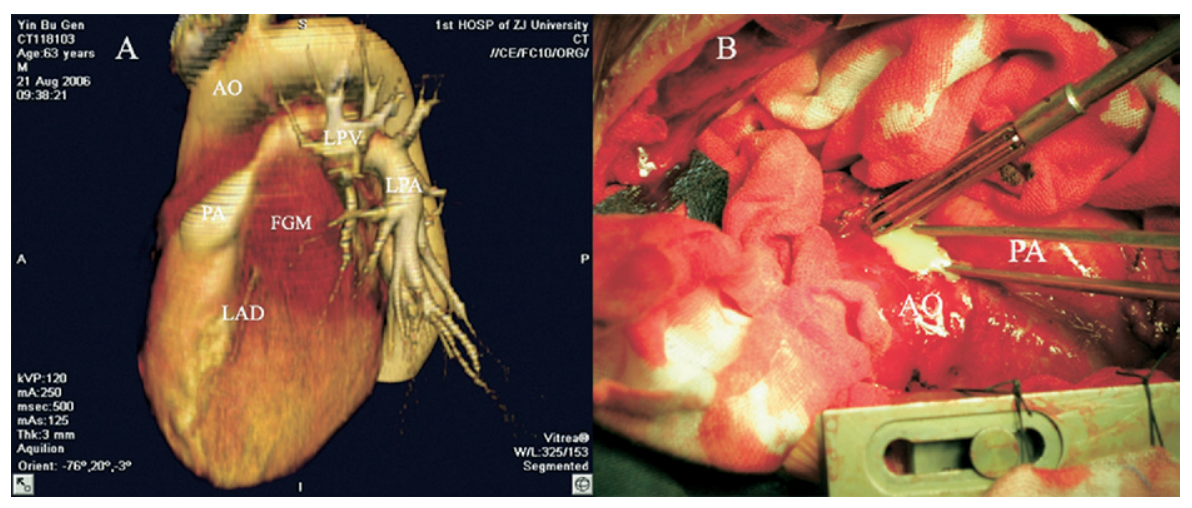

Figure 2. A, The mediastinal mass with 3-dimension (B) caseous necrosis flowing out of the mass from the incison during the operation. AO, Aorta; $P A$, pulmonary artery; $L P A$, left pulmonary artery; $L P V$, left pulmonary vein; FGM, fibrosing granulomatous mass; $L A D$, left anterior descending.

manifestation of mediastinal granuloma. Chronic granulomatous inflammation is characterized by slow progression of fibrosis and fibrocaseous granulomas that develop in the regional mediastinal lymph nodes. ${ }^{2}$ Tuberculosis is thought to be the precursor of this pathologic condition with significantly less frequency. Many mediastinal granulomas are asymptomatic, but others may produce nonspecific signs and symptoms including cough, dyspnea, and wheezing. The inflammatory process that develops in the transverse sinus of the mediastinum is very uncommon. The inflammation is usually associated with considerable fibrosis. The hallmark of a mediastinal granuloma is the formation of an encapsulated fibrous mass surrounding a core of caseating lymph nodes. The development of symptoms and signs in granulomatous mediastinitis depends on the degree of fibrotic reaction around the mediastinal lymph nodes. The most common complications of mediastinal granuloma are fibrosing mediastinitis and obstruction of the superior vena cava. The appearance may mimic a malignant process. $^{3}$
The indication for surgery is to establish the diagnosis. In our case, surgery was required to relieve severe compression and confirm the diagnosis. In some severe compression syndromes, surgery cannot be performed because the operative risk would be too high. Once the diagnosis has been obtained, appropriate therapy can be commenced. Our case can be considered unique because of the giant dimensions and rare location of the fibrosing granulomatous mediastinitis of tuberculosis.

\section{References}

1. Ramakastan R, Shah P. Dysphagia due to mediastinal fibrosis in advanced pulmonary tuberculosis. Am J Roentgenol. 1990;154:61-3.

2. Dines D, Payne WS, Bernatz PE, Pailero PC. Mediastinal granuloma and fibrosing mediastinitis. Chest. 1979;75:320-4.

3. Bays S, Rajakaruna C, Sheffield E, Morgan A. Fibrosing mediastinitis as a cause of superior vena cava syndrome. Eur J Cardiothorac Surg. 2004;26:453-5. 